Portland State University

PDXScholar

$11-18-2021$

\title{
Geometric Characterization of Continuously Defective Elastic Crystals
}

Marek Z. Elźanowski

Portland State University, elzanowskim@pdx.edu

Follow this and additional works at: https://pdxscholar.library.pdx.edu/mth_fac

Part of the Physical Sciences and Mathematics Commons

Let us know how access to this document benefits you.

Citation Details

Elżanowski, M. Z. (2021). Geometric characterization of continuously defective elastic crystals.

Mathematics and Mechanics of Solids. https://doi.org/10.1177/10812865211058769

This Citation is brought to you for free and open access. It has been accepted for inclusion in Mathematics and Statistics Faculty Publications and Presentations by an authorized administrator of PDXScholar. Please contact us if we can make this document more accessible: pdxscholar@pdx.edu. 


\title{
Geometric characterization of continuously defective elastic crystals
}

Mathematics and Mechanics of Solids $1-10$

(C) The Author(s) 2021

Article reuse guidelines:

sagepub.com/journals-permissions DOI: $10.1|77 / 108| 2865211058769$ journals.sagepub.com/home/mms

\section{(SSAGE}

\author{
Marek Z. Elżanowski \\ Fariborz Maseeh Department of Mathematics and Statistics, \\ Portland State University, Portland, OR, USA \\ School of Mathematical and Statistical Sciences, Arizona State University, Tempe, AZ, USA
}

Received 19 August 2021; accepted: 21 October 2021

\begin{abstract}
We show how some differential geometric structures associated with a concept of a homogeneous space appear naturally in a kinematic model of continuously distributed defects in an elastic crystal solid and discuss how one can use them to describe the defectiveness of such a continuum.
\end{abstract}

\section{Keywords}

Continuous distributions of defects, elastic crystals, homogeneous spaces, invariant connections, curvature

\section{Introduction}

One of the fundamental questions of the kinematic model of defective elastic crystals proposed by Davini [1] is to identify quantities which properly characterize the defectiveness of a crystal and which can also be useful in phenomenological approaches describing inelastic behavior of continuum bodies. Assuming that a kinematic state of a continuous crystal is determined by three linearly independent vector fields (a lattice) defined over an open region in $\mathbb{R}^{3}$, a non-commutativity of pairs of these vector fields is considered a sign of a presence of defects. The said vector fields are viewed as obtained from the underlaying discrete atomic structure by some averaging process and are expected to be invariant under elastic deformations of a continuum. Respectively, the objects which characterize locally such a collection of vector fields are also expected to be elastically invariant. One such object, which can be viewed as a first-order measure of defectiveness at a point of a lattice, is the dislocation density tensor [1] (see Equation (21)).

When the dislocation density tensor is a constant function of position, thus defining a uniformly defective state of a continuous crystal, the underlying space can be identified with a Lie group acting on itself and the theory of Lie groups and algebras is used to study such states [2]. However, when the dislocation density tensor is a non-trivial function of a material point, this identification is no longer available. That is, even though the underlying space cannot be identified with a Lie group, there still exists a Lie group (of dimension higher than three) acting on $\mathbb{R}^{3}$ in a manner consistent with the lattice vector fields [3]. This allows us to equip $\mathbb{R}^{3}$ with a structure of a differentiable homogeneous space and use the notion of a canonical linear connection and, in particular, its curvature as a second-order measure of defectiveness of a crystal state. ${ }^{1}$

Starting from a perspective of the uniformly defective states, we focus in our presentation on states which are non-uniformly defective and, in particular, canonically reductive (26), as other states are not fully amenable 
to this approach. After the first section in which we present the general differential geometric framework associated with non-uniformly defective states, we introduce in Section 2 the concept of a lattice connection, the torsion of which relates to the dislocation density tensor. In Section 3, we discuss the concept of a lattice canonical connection available for non-uniformly defective crystal states which are also reductive. In Section 4, we introduce the concept of a canonically reductive state and show how the curvature of the corresponding lattice canonical connection relates to the derivatives of the torsion of the lattice connection. We conclude the paper by discussing two different examples of continuous lattices, one which is canonically reductive and one which is not.

The work reported in this paper is an extension and a continuation of our research presented in $[5,6]$.

\section{Geometric framework}

As was stated in the introduction, we postulate that a kinematic state of a continuous distribution of defects in a solid is defined by a lattice, ${ }^{2}$ that is, $n$ linearly independent smooth vector fields $\mathfrak{l}=\left\{l_{1}, \ldots, l_{n}\right\}$ defined on an open subset $U \subset \mathbb{R}^{n}$, where, in most applications, $n=2,3{ }^{3}$ We postulate that the vector fields $l_{1}, \ldots, l_{n}$ generate a finite-dimensional ${ }^{4}$ (complete) Lie subalgebra $\mathbf{L}$ of the algebra of all smooth vector fields on $U$. We call this algebra the lattice algebra of the continuous lattice $\mathfrak{l}$. This, in turn, implies the existence of an abstract Lie group $G$ acting smoothly on the body $U$ on the left and such that its (left) Lie algebra $\mathfrak{g}$ is isomorphic to the lattice algebra $\mathbf{L}$ (see [7]). Let a smooth mapping

$$
\phi: G \times U \rightarrow U
$$

represent the said action of $G$ on $U$ satisfying a condition that

$$
\phi\left(g_{1} g_{2}, p\right)=\phi\left(g_{1}, \phi\left(g_{2}, p\right)\right)
$$

for every $p \in U$ and every pair $g_{1}, g_{2} \in G$, where $g_{1} g_{2}$ denotes a group multiplication in $G$. We postulate that the action $\phi$ is transitive on $U$ implying that the orbit map

$$
\phi_{p}=\phi(\cdot, p): G \rightarrow U, p \in U,
$$

is onto and that its tangent map

$$
d \phi_{p}: T G \rightarrow T U,
$$

where $T G$ and $T U$ denote the corresponding tangent spaces, establishes an isomorphism between the (right) Lie algebra ${ }^{5}$ of $G$ and the lattice algebra $\mathbf{L}$ (see [8]).

Select a point, say, $p_{0} \in U$. The isotropy group of the action $\phi$ at $p_{0}$

$$
G_{0}=\left\{g \in G: \phi\left(g, p_{0}\right)=p_{0}\right\}
$$

is a closed subgroup of the group $G$. It depends, in general, on the choice of a point $p_{0} \in U$. However, owing to the transitivity of the action $\phi$, the isotropy groups at different points are conjugate to each other, thus, isomorphic.

Given an isotropy group $G_{0}$ at a point $p_{0} \in U$, one can show that the underlying space $U$ is a homogeneous space, that is, it is diffeomorphically equivalent to the left quotient $G \backslash G_{0}$. This equivalence is established by a map $\Phi: G \backslash G_{0} \rightarrow U$ such that

$$
\Phi\left(g G_{0}\right)=\phi\left(g, p_{0}\right),
$$

where $g G_{0}=\left\{g h: h \in G_{0}\right\}$ denotes a (left) coset of the isotropy group $G_{0}$ generated by an element $g \in G$. Not only the quotient $G \backslash G_{0}$ is diffeomorphic to the space $U$, but the group $G$ acts on it on the left mimicking the corresponding left action of $\phi$ on $U$. Namely,

$$
\phi\left(g_{1}, \Phi\left(g_{2} G_{0}\right)\right)=\phi\left(g_{1}\left(\phi\left(g_{2}, p_{0}\right)\right)=\phi\left(g_{1} g_{2}, p_{0}\right)=\Phi\left(\left(g_{1} g_{2}\right) G_{0}\right) .\right.
$$

Moreover, looking at this construction from a different angle, we observe that the left action $\phi$ of $G$ on the space $U$ defines a principal $G_{0}$-bundle over $U$ with the group $G$ as its total space, the isotropy group $G_{0}$ as its structure 
group, and the projection $\pi: G \rightarrow U$ such that $\pi(g)=\phi\left(g, p_{0}\right)$. Indeed, the projection $\pi$ is a differentiable mapping and the isotropy group $G_{0}$ acts freely on $G$ on the right preserving individual fibers, i.e., given $h \in G_{0}$

$$
\pi(g h)=\phi\left(g h, p_{0}\right)=\phi\left(g, \phi\left(h, p_{0}\right)\right)=\phi\left(g, p_{0}\right)=\pi(g)
$$

for every $g \in G$ and every $h \in G_{0}$. Note that the fibers of the principal bundle $\pi: G \rightarrow U$ are the cosets of the quotient $G \backslash G_{0}$.

In addition, it can be shown [9] (see also [10]) that the bundle $\pi: G \rightarrow U$ is isomorphic to a subbundle of the bundle of linear frames on $U$. To this end, given $g \in G$, consider a mapping $\phi_{g}: U \rightarrow U$ such that $\phi_{g}(p)=\phi(g, p)$ for every $p \in U$. When $g$ is an element of the isotropy group $G_{0}$ at $p_{0}$ the tangent map $d_{p_{0}} \phi_{g}: T_{p_{0}} U \rightarrow T_{p_{0}} U$ is a linear automorphism. Selecting a frame at $p_{0}$, that is a linear isomorphism $u_{0}: \mathbb{R}^{n} \rightarrow T_{p_{0}} U$ assigning coordinates to a vector in $T_{p_{0}} U$, we are able to construct a group homomorphism $\lambda: G_{0} \rightarrow G L(n, \mathbb{R})$ such that

$$
\lambda(h)=u_{0}^{-1} \circ d_{p_{0}} \phi_{h} \circ u_{0},
$$

for any $h \in G_{0}$. The mapping $\lambda$ is known as a linear isotropy representation of $G_{0}$ in the general linear group of $\mathbb{R}^{n}$ (see, e.g., [9]). Consequently, the collection of mappings

$$
L\left(U, G_{0}\right)=\left\{d_{p_{0}} \phi_{g} \circ u_{0}: \mathbb{R}^{n} \rightarrow U: g \in G\right\}
$$

is a reduction of the bundle of linear frames of $U$ to the linear isotropy group $\lambda\left(G_{0}\right) \subset G L(n, \mathbb{R})$. The mapping $f: G \rightarrow L\left(U, G_{0}\right)$ assigning to $g \in G$ a linear frame $d_{p_{0}} \phi_{g} \circ u_{0}$ is a principal bundle isomorphism over the identity map on $U \subset \mathbb{R}^{n}$. In particular, for any $g \in G$ and $h \in G_{0}$

$$
f(g h)=d_{p_{0}} \phi_{g h} \circ u_{0}=d_{p_{0}} \phi_{g} \circ u_{0} \circ \lambda(h)=f(g) \lambda(h) .
$$

Example 1. To illustrate how the geometric structures introduced so far appear in a concrete situation let us consider, using the standard cartesian coordinate system in $\mathbb{R}^{2}$, a two-dimensional lattice

$$
\mathfrak{l}=\left\{l_{1}, l_{2}\right\}=\left\{\mathbf{e}_{1},-x \mathbf{e}_{2}\right\}
$$

where the vectors $\mathbf{e}_{1}, \mathbf{e}_{2}$ denote the corresponding unit basis. As the bracket $l_{3}=\left[l_{1}, l_{2}\right]=-\mathbf{e}_{2}$ and as $\left[l_{1}, l_{3}\right]=$ $\left[l_{2}, l_{3}\right]=0$ the given lattice generates a three-dimensional Lie algebra $\mathbf{L}=\operatorname{span}\left\{l_{1}, l_{2}, l_{3}\right\}$. Elementary calculations show that the action of the corresponding three-parameter Lie group $G$ on $U=\left\{(x, y) \in \mathbb{R}^{2}: x \neq 0\right\}$ is given by the mapping $\phi: G \times U \rightarrow U$ such that

$$
\phi((a, b, c),(x, y))=(x+a, y-(x+a) b-c),
$$

where a triple $(a, b, c)$ represents a group element and where $(x, y) \in U$. Enforcing the requirement that the group $G$ acts on the left, we obtain the group multiplication in $G$ as

$$
(a, b, c)(\bar{a}, \bar{b}, \bar{c})=(a+\bar{a}, b+\bar{b}, c+\bar{c}-a \bar{b}),
$$

for any two elements $(a, b, c),(\bar{a}, \bar{b}, \bar{c}) \in G$.

$A$ one-parameter isotropy group of the action $\phi$ at a point $p_{0}=\left(x_{0}, y_{0}\right) \in U$ is

$$
G_{0}=\left\{\left(0, b,-x_{0} b\right): b \in \mathbb{R}\right\} .
$$

It is easy to see that the projection $\pi((a, b, c))=\phi\left((a, b, c),\left(x_{0}, y_{0}\right)\right)$ is such that $\pi\left(G_{0}\right)=\left(x_{0}, y_{0}\right)$. Finally, the linear isotropy representation (in the standard frame) is given by

$$
\lambda(h)=\left(\begin{array}{cc}
1 & 0 \\
-b & 1
\end{array}\right)
$$

for every $h \in G_{0}$. Note that the image

$$
\lambda\left(G_{0}\right)=\left\{\left(\begin{array}{cc}
1 & 0 \\
-b & 1
\end{array}\right): b \in \mathbb{R}\right\}
$$

is indeed a subgroup of $G L(2, \mathbb{R})$. 


\section{Lattice connection}

The fact that one can associate a specific homogeneous space with a continuous lattice allows us to identify a set of (measurable) geometric characteristics which may be used to describe defectiveness of a kinematic state of a continuum. Namely, given a lattice $\mathfrak{l}$ on $U \subset \mathbb{R}^{n}$ and the corresponding Lie group $G$ acting on $U$, there exist (subject to some additional assumptions) two linear connections, the torsions and the curvatures of which may be interpreted as characterizing the defectiveness of a kinematic state.

First, given a lattice $\mathfrak{l}=\left\{l_{1}, \ldots, l_{n}\right\}$, the linearly independent vector fields $l_{i}, i=1, \ldots, n$, define on $U$ a long-distant parallelism and a flat linear connection, called a lattice connection, associated with it. Its Christoffel symbols $\Gamma_{j k}^{i}, i, j, k=1, \ldots, n$, are

$$
\Gamma_{j k}^{i}=-\left(l_{j}^{m}\right)^{-1} \frac{\partial l_{m}^{i}}{\partial x_{k}}
$$

where the matrices $l_{m}^{i}, i, m=1, \ldots, n$, represent coordinates of the vectors defining the lattice in the standard coordinate system $x_{1}, \ldots, x_{n}$ on $U$ (see, e.g., [11]). Although the curvature of a lattice connection vanishes its torsion

$$
T_{j k}^{i}=\Gamma_{[j k]}^{i}
$$

does not, in general, and it is often accepted (see Remark 1) as representing the defectiveness of a given lattice $\mathfrak{l}$. Note that the components of a torsion of the lattice connection can also be given in terms of the Lie brackets of the algebra $\mathbf{L}$ as

$$
\left[l_{j}, l_{k}\right]=T_{j k}^{i} l_{i},
$$

where the summation convention over repeated indices is enforced.

Remark 1. The archetypical object associated with a defectiveness of a lattice in dimension $n=3$ (see [1]) is the dislocation density tensor $S_{i j}$ defined in terms of the dual lattice $\left\{\eta_{1}, \eta_{2}, \eta_{3}\right\}$ such that $l_{i} \cdot \eta_{j}=\delta_{i j}$, where $\delta_{i j}$ denotes the usual Kronecker delta. That is, the components $S_{i j}$ of the dislocation density tensor are such that

$$
n(p) S^{i j}(p)=\nabla \wedge \eta^{i}(p) \cdot \eta^{j}(p), \quad i, j=1,2,3, p \in M,
$$

where $n(p)$ denotes the lattice volume element (the determinant of the dual lattice) and where the objects $\mathbf{b}^{i}=$ $\nabla \wedge \eta^{i}(p)$ are known in the material science literature as the Burgers vectors representing a distortion of a lattice cased by a presence of defects [12, 13]. It can be shown [14] that the dislocation density tensor $S_{i j}$ and the torsion $T_{j k}^{i}$ are related by

$$
T_{j k}^{i}=\epsilon_{r j k} S^{i r}
$$

where $\epsilon_{r j k}$ is the alternating tensor.

When investigating possible dislocated states and the lattices representing them one may consider the following three scenarios.

(A) First, if the torsion of a (flat) lattice connection $\Gamma_{j k}^{i}$ vanishes the connection is trivial and the lattice vector fields $l_{i}, i=1, \ldots, n$, defining the corresponding long-distance parallelism commute. The lattice $\mathfrak{l}$ is holonomic and the lattice Lie algebra $\mathbf{L}$ is abelian. Physically, the kinematic state the lattice $\mathbf{I}$ represents is homogeneous, that is, no defects are present and, in dimension three, the dislocation density tensor $S^{i j}$ vanishes identically. The group $G$ the algebra $\mathbf{L}$ induces can be identify, without loss of generality, with $\mathbb{R}^{n}$ acting on $U$ by translations as there exists always, at least locally, a coordinate system, say, $x_{1}, \ldots, x_{n}$ on $U$ such that $\mathfrak{l}=\left\{\frac{\partial}{\partial x_{1}}, \ldots, \frac{\partial}{\partial x_{n}}\right\}$. In other words, the kinematic state the lattice $\mathfrak{l}$ represents is invariant under translations.

(B) Second, assume that the torsion of a lattice connection does not vanish but its value is base point independent. This implies that the components of the torsion tensor $T_{j k}^{i}$ are simply the Lie algebra constants of the lattice algebra $\mathbf{L}$ and (in dimension three) the dislocation density tensor is constant. The Lie group $G$, still viewed as $\mathbb{R}^{n}$, acts on $U$ in a non-trivial way. We say that such a kinematic state is uniformly defective which means that open neighborhoods of different material points are diffeomorphically equivalent. As such diffeomorphisms are viewed as elastic deformations [2], being uniformly defective means being locally elastically related. 
Example 2. Consider, using a cartesian coordinate system in $\mathbb{R}^{3}$, a lattice

$$
\mathfrak{l}=\left\{l_{1}, l_{2}, l_{3}\right\}=\left\{\mathbf{e}_{1}, \mathbf{e}_{2}, x \mathbf{e}_{1}+y \mathbf{e}_{2}+\mathbf{e}_{3}\right\}
$$

defined on $U=\left\{(x, y, z) \in \mathbb{R}^{3}: x>0, y>0\right\}$. As $\left[l_{1}, l_{3}\right]=l_{1},\left[l_{2}, l_{3}\right]=l_{2}$, and $\left[l_{1}, l_{2}\right]=0$ the only nonzero torsion coefficients are $T_{13}^{1}=T_{23}^{2}=1$. The corresponding Burgers vectors are $\mathbf{b}^{1}=\mathbf{e}_{2}, \mathbf{b}^{2}=-\mathbf{e}_{1}$, and $\mathbf{b}^{3}=\mathbf{0}$, and the dislocation density tensor

$$
S_{i j}=\left(\begin{array}{ccc}
0 & 1 & 0 \\
-1 & 0 & 0 \\
0 & 0 & 0
\end{array}\right) .
$$

(C) Finally, suppose that a lattice $\mathfrak{l}$ is such that some components of the torsion $T_{j k}^{i}$ of the lattice connection $\Gamma_{j k}^{i}$ are non-trivial functions of position. ${ }^{6}$ The fact that the torsion tensor is material-point-dependent implies that the lattice algebra $\mathbf{L}$ is of a finite dimension, ${ }^{7}$ say $m$, higher than the dimension of the base space $U$, and the kinematic state $\mathfrak{l}$ represents is said to be non-uniformly defective. The lattice algebra $\mathbf{L}$ induces an $m$-parameter connected Lie group $G$ acting on $U$ in such a way that the isotropy group $G_{0}$ is non-trivial and of dimension $m-n \geq 1$. As the isotropy group is a Lie subgroup of the Lie group $G$ its (left) Lie algebra $\mathfrak{g}_{0}$ is a Lie subalgebra of the (left) Lie algebra $\mathfrak{g}$. Viewing $\mathfrak{g}$ as a vector space of all left-invariant vector fields on $G$, it can always be represented as a simple sum of the isotropy algebra $\mathfrak{g}_{0}$ and a vector space complement $\mathbf{V} \subsetneq \mathfrak{g}$. In other words,

$$
\mathfrak{g}=\mathfrak{g}_{0} \oplus \mathbf{V}
$$

for some vector subspace $\mathbf{V}$ of the algebra $\mathfrak{g}$ viewed as a vector space. Note that $\mathbf{V}$ is not uniquely defined and that, in general, it is not a Lie subalgebra of $\mathfrak{g}$.

In the next section, we look closer at possible characteristics of non-uniformly defective states.

\section{Lattice canonical connection}

Consider a lattice $\mathfrak{l}=\left\{l_{1}, \ldots, l_{n}\right\}$ representing a non-uniformly defective kinematic state of a solid $U$. As we pointed out earlier, this means that its lattice algebra $\mathbf{L}$ is of dimension higher than the dimension of $U$ and that the corresponding Lie group $G$ acting on $U$ on the left has a non-trivial isotropy group $G_{0}$, or its conjugate, at any and all points of $U$. This also means that the left Lie algebra $\mathfrak{g}$ of $G$ can be represented as $\mathfrak{g}=\mathfrak{g}_{0} \oplus \mathbf{V}$ for some $n$-dimensional vector space $\mathbf{V} \subset \mathfrak{g}$ of left-invariant vector fields on $G$. As the choice of a subspace $\mathbf{V}$ is not unique, we further assume that one can select a space $\mathbf{V}$ such that the decomposition $\mathfrak{g}_{0} \oplus \mathbf{V}$ is reductive [9], that is,

$$
\left[\mathfrak{g}_{0}, \mathbf{V}\right] \subseteq \mathbf{V}
$$

We should point out here that although given a subalgebra $\mathfrak{g}_{0} \subsetneq \mathfrak{g}$ there is always a vector space $\mathbf{V} \subseteq \mathfrak{g}$ such that $\mathfrak{g}=\mathfrak{g}_{0} \oplus \mathbf{V}$, not every such decomposition is reductive. In fact, given $\mathfrak{g}$ there may not exist a vector complement $\mathbf{V}$ making the decomposition $\mathfrak{g}=\mathfrak{g}_{0} \oplus \mathbf{V}$ reductive [15].

Every vector space complement $\mathbf{V}$ forms a horizontal distribution on the principal bundle $\pi: G \rightarrow U$ in the sense that it depends smoothly on $G$ and the tangent map $d \pi: T G \rightarrow T U$ is surjective with the subalgebra $\mathfrak{g}_{0}$ as its kernel. However, only if the decomposition $\mathfrak{g}_{0} \oplus \mathbf{V}$ is reductive, $\mathbf{V}$ defines a horizontal distribution of a left-invariant principal bundle connection on $\pi: G \rightarrow U$. Indeed, as we showed in [6], the fact that $\left[\mathfrak{g}_{0}, \mathbf{V}\right] \subseteq \mathbf{V}$ implies that $\mathbf{V}$ is invariant under the right action of the isotropy group (see also [16]). Such a left-invariant principal bundle connection is called a canonical connection on the homogeneous space $U \cong G \backslash G_{0}{ }^{8}$

As the bundles $\pi: G \rightarrow U$ and $L\left(U, G_{0}\right)$ are isomorphic, the canonical connection associated with the distribution $\mathfrak{g}_{0} \oplus \mathbf{V}$ induces a linear connection on $U$, called a linear canonical connection $[9,16]$.

Theorem 1. Let $\mathbf{I}$ be continuous lattice defined on a body manifold U. Assume that the corresponding homogenous space $G \backslash G_{0}$ admits a reductive decomposition $\mathfrak{g}=\mathfrak{g}_{0} \oplus \mathbf{V}$ for some vector complement $\mathbf{V} \subset \mathfrak{g}$, where $G_{0}$ is an isotropy group of the left action of $G$ on $U$ evaluated at $p_{0} \in U$. Then, relative to the choice of the frame $u_{0}: \mathbb{R}^{n} \rightarrow T_{p_{0}} U$, the torsion and curvature of the corresponding (left-invariant) linear canonical connection are given at $p_{0}$ by: 
(a) $\widehat{T}(X, Y)=-[X, Y]_{\mathbf{V}}$;

(b) $\widehat{R}(X, Y) Z=-\left[[X, Y]_{\mathfrak{g}_{0}}, Z\right]$;

for any left-invariant vector field $X, Y, Z \in \mathbf{V}$, where $[\cdot, \cdot]_{\mathbf{V}}$ and $[\cdot, \cdot]_{\mathfrak{g}_{0}}$ denote $\mathbf{V}$ and $\mathfrak{g}_{0}$ components, respectively, of the Lie algebra bracket in $\mathfrak{g}$. In addition, both tensors are left-invariant, thus covariantly constant.

Remark 2. It seems appropriate now to make a few comments regarding the existence of a linear canonical connection induced by a lattice $\mathfrak{l}$.

First, we would like to point out that although the concept of a linear canonical connection was introduced in the context of a non-uniformly defective continuous lattice, it existence is also guaranteed when the lattice is uniformly defective. That is, when a lattice $\mathfrak{l}$ represents a uniformly defective kinematic state, its lattice Lie algebra $\mathbf{L}$ is of the same dimension as the body $U$ and the corresponding isotropy group is trivial. This implies that any horizontal distribution $\mathbf{V}$ is identical to the left Lie algebra of the group G. The linear canonical connection is unique and equal to the lattice connection, thus providing no addition geometric characteristics of the lattice $\mathrm{l}$.

However, when a lattice frame $\mathfrak{l}$ represents truly non-uniformly defective state, the existence of a linear canonical connection cannot be guaranteed a priori. Indeed, as we pointed out earlier, given a continuous lattice and the corresponding lattice algebra $\mathbf{L}$, the induced homogenous space $G / G_{0}$ may or may not be reductive. If it is not reductive, the concept of a linear canonical connection is not well defined. However, even if it is reductive the choice of the corresponding vector complement $\mathbf{V}$ may not be unique as there may exist a number of different vector subspaces $\mathbf{V}$ of $\mathfrak{g}$ such that the $\left[\mathfrak{g}_{0}, \mathbf{V}\right] \subseteq \mathbf{V}$, thus inducing different linear canonical connections. Although all such connections characterize in some ways the underlying defective crystal structure not all seem to provide useful geometric characteristics of it, the issue which we discuss in the remainder of this paper.

Example 3. Let us revisit once again Example 1. Using (14) defining the group multiplication in G, we obtain that the left Lie algebra

$$
\mathfrak{g}=\operatorname{span}\left\{v_{1}, v_{2}, v_{3}\right\}=\operatorname{span}\left\{\mathbf{g}_{1}, \mathbf{g}_{2}-a \mathbf{g}_{3}, \mathbf{g}_{3}\right\}
$$

and that the left-invariant vector field generating the isotropy algebra $\mathfrak{g}_{0}$ is

$$
v_{0}=\mathbf{g}_{2}-\left(x_{0}+a\right) \mathbf{g}_{3} .
$$

Selecting the space $\mathbf{V}=\operatorname{span}\left\{v_{1}, v_{3}\right\}$, we obtain a reductive decomposition $\mathfrak{g}=\mathfrak{g}_{0} \oplus \mathbf{V}$ as $\left[v_{1}, v_{0}\right]=-v_{3} \in \mathbf{V}$ and $\left[v_{3}, v_{0}\right]=0$. However, the corresponding linear canonical connection is trivial as due to the fact that $\mathbf{V}$ is an abelian subalgebra of $\mathfrak{g}$ both curvature and torsion vanish. We discuss other choices of $\mathbf{V}$ in the next section.

\section{Curvature of a non-uniformly defective state}

Given a continuous lattice $\mathfrak{l}$ representing a non-uniformly defective state, as signified by a non-constant torsion $T_{j k}^{i}$ of its lattice connection, it is only natural to determine whether there exists any relation between this characteristic of a lattice and the form of the torsion and curvature tensors of the corresponding linear canonical connection (Theorem 1), if it exists. To this end, given a lattice $\mathbf{l}$, defined by the vector fields $l_{i}, i=1, \ldots, n$, let $w_{1}, \ldots, w_{n}$ denote right-invariant vector fields on $G$ such that

$$
d_{g} \phi_{p_{0}}\left(w_{i}\right)=l_{i}, \quad i=1, \ldots, n .
$$

As the tangent mapping $d_{g} \phi_{p_{0}}(4)$ is of maximum rank and as the vector fields $l_{1}, \ldots, l_{n}$ are linearly independent, the right-invariant vector fields $w_{1}, \ldots, w_{n}$ always exist and are linearly independent. Let $v_{1}, \ldots, v_{n}$ be the equivalent set of left-invariant vector fields on $G$, that is, a set of the elements of the algebra $\mathfrak{g}$ such that

$$
v_{i}=d i\left(w_{i}\right), \quad i=1, \ldots, n,
$$

where $i$ is the inverse map on the group $G$. It is easy to show [8] that

$$
\left[v_{i}, v_{j}\right]=-\left[w_{i}, w_{j}\right], \quad\left[v_{i}, w_{j}\right]=0, \quad i, j=1, \ldots, n .
$$

Define a canonical vector space $\mathbf{V}_{c}=\operatorname{span}\left\{v_{1}, \ldots, v_{n}\right\}$. It is clear that $\mathbf{V}_{c}$ is a vector subspace of the Lie algebra $\mathfrak{g}$ and that $\mathfrak{g}=\mathfrak{g}_{0} \oplus \mathbf{V}_{c}$ as the isotropy subalgebra $\mathfrak{g}_{0}$ is the kernel of the projection $d \pi: T G \rightarrow T U$. Assume 
that the lattice $\mathbf{I}$ is such that the decomposition $\mathfrak{g}=\mathfrak{g}_{0} \oplus \mathbf{V}_{c}$ is reductive, a property which cannot be guaranteed in general. We shall call the corresponding linear connection on $L\left(G_{0}, u\right)$ the lattice canonical connection ${ }^{9}$ of a continuous lattice $\mathbf{l}$.

The coefficients of the torsion tensor of the lattice canonical connection (in the frame $v_{1}, \ldots, v_{n}$ ) are the smooth functions $-\widehat{T}_{j k}^{i}: U \rightarrow \mathbb{R}, i, j, k=1, \ldots, n$, such that

$$
\widehat{T}_{j k}^{i} v_{i}=\left[v_{j}, v_{k}\right] \mathbf{v}_{c},
$$

where $[\cdot, \cdot]_{\mathbf{V}_{c}}$ denotes a $\mathbf{V}_{c}$ component of the Lie bracket of the algebra $\mathfrak{g}$ and where the minus sign is selected for convenience. Respectively, the coefficients of the curvature tensor of the lattice canonical connection are the smooth functions $-\widehat{R}_{j k l}^{i}: U \rightarrow \mathbb{R}$ such that

$$
\widehat{R}_{j k l}^{i} v_{i}=\left[\left[v_{j}, v_{k}\right]_{\mathfrak{g}_{0}}, v_{l}\right],
$$

where $[\cdot, \cdot]_{\mathfrak{g}_{0}}$ is a $\mathfrak{g}_{0}$ component of the Lie bracket in $\mathfrak{g}$.

Theorem 1 and the relation (31) allow us to show the relation between the torsion $\widehat{T}_{j k}^{i}$ and $T_{j k}^{i}$.

Corollary 1. Assume that the continuous lattice $\mathbf{l}=\left\{l_{1}, \ldots, l_{n}\right\}$ admits a lattice canonical connection corresponding to a reductive decomposition $\mathfrak{g}_{0} \oplus \mathbf{V}_{c}$. Then, the torsion coefficients of the lattice canonical connection in the equivalent frame $v_{i}, i=1, \ldots, n$ are identical to the torsion coefficients of the lattice connection $\Gamma_{j k}^{i}(18)$, that is,

$$
\widehat{T}_{j k}^{i}=-T_{j k}^{i}\left(p_{0}\right)
$$

Furthermore, consider the Jacoby identity of the Lie algebra $\mathfrak{g}$ applied to the canonical (left-invariant) vector fields $v_{i} \in \mathbf{V}_{c}$ (30), namely

$$
\left[\left[v_{j}, v_{k}\right], v_{l}\right]+\left[\left[v_{k}, v_{l}\right], v_{j}\right]+\left[\left[v_{l}, v_{j}\right], v_{k}\right]=0, j, k, l=1, \ldots, n .
$$

Invoking the reductive decomposition $\mathfrak{g}=\mathfrak{g}_{0} \oplus \mathbf{V}_{c}$ and the definition of a torsion and a curvature of a linear canonical connection (32) and (33), we obtain that, for example,

$$
\begin{aligned}
{\left[\left[v_{j}, v_{k}\right], v_{l}\right] } & =\left[\left[v_{j}, v_{k}\right]_{\mathbf{v}}, v_{l}\right]+\left[\left[v_{j}, v_{k}\right]_{\mathfrak{g}_{0}}, v_{l}\right] \\
& =\left[\widehat{T}_{j k}^{i} v_{i}, v_{l}\right]+\widehat{R}_{j k l}^{m} v_{m} \\
& =\widehat{T}_{j k}^{i}\left[v_{i}, v_{l}\right]+\widehat{R}_{j k l}^{m} v_{m} \\
& =\widehat{T}_{j k}^{i}\left[v_{i}, v_{l}\right]_{\mathbf{v}}+\widehat{T}_{j k}^{i}\left[v_{i}, v_{l}\right]_{\mathfrak{g}_{0}}+\widehat{R}_{j k l}^{m} v_{m} \\
& =\widehat{T}_{j k}^{i} \widehat{T}_{i l}^{p} v_{p}+\widehat{T}_{j k}^{i}\left[v_{i}, v_{l}\right]_{\mathfrak{g}_{0}}+\widehat{R}_{j k l}^{m} v_{m} .
\end{aligned}
$$

The other two terms of the Jacoby identity can be represented the same way. Adding all three terms and realizing that the sum of all $\mathfrak{g}_{0}$-terms vanishes, we obtain a set of $n$ equations for the coefficient of the $\mathbf{V}_{c}$-part of the identity:

$$
\widehat{T}_{j k}^{i} \widehat{T}_{i l}^{m}+\widehat{T}_{k l}^{r} \widehat{T}_{r j}^{m}+\widehat{T}_{l j}^{p} \widehat{T}_{p k}^{m}+\widehat{R}_{j k l}^{m}+\widehat{R}_{k l j}^{m}+\widehat{R}_{l j k}^{m}=0, \quad m=1, \ldots, n .
$$

Subsequently, Corollary 1 implies that

$$
\widehat{R}_{j k l}^{m}+\widehat{R}_{k l j}^{m}+\widehat{R}_{l j k}^{m}=-\left[T_{j k}^{i} T_{i l}^{m}+T_{k l}^{r} T_{r j}^{m}+T_{l j}^{p} T_{p k}^{m}\right]\left(p_{0}\right) .
$$

Looking back at the lattice $\mathfrak{l}=\left\{l_{1}, \ldots, l_{n}\right\}$ and the Jacoby identity of the algebra $\mathbf{L}$ we have

$$
\begin{aligned}
& {\left[\left[l_{j}, l_{k}\right], l_{l}\right]+\left[\left[l_{k}, l_{l}\right], l_{j}\right]+\left[\left[l_{l}, l_{j}\right], l_{k}\right]} \\
& =\left[T_{j k}^{a} l_{a}, l_{l}\right]+\left[T_{k l}^{b} l_{b}, l_{j}\right]+\left[T_{l j}^{c} l_{c}, l_{k}\right] \\
& =T_{j k, l}^{a} l_{a}+T_{j k}^{a}\left[l_{a}, l_{l}\right]+T_{k l, j}^{b} l_{b}+T_{k l}^{b}\left[l_{b}, l_{j}\right]+T_{l j, k}^{c} l_{c}+T_{l j}^{c}\left[l_{c}, l_{k}\right] \\
& =T_{j k, l}^{a} l_{a}+T_{j k}^{a} T_{a l}^{d} l_{d}+T_{k l, j}^{b} l_{b}+T_{k l}^{b} T_{b j}^{e} l_{e}+T_{l j, k}^{c} l_{c}+T_{l j}^{c} T_{c k}^{f} l_{f}=0
\end{aligned}
$$

where a comma denotes a directional derivative in the direction of a specific vector field. Comparing this with (38), we are finally able to show how the curvature of a lattice canonical connection (33) relates to the derivatives of the torsion tensor $T_{j k}^{i}$ of the lattice connection (18). 
Corollary 2. Suppose a continuous lattice $\mathbf{I}=\left\{l_{1}, \ldots, l_{n}\right\}$ admits a lattice canonical connection corresponding to a reductive (canonical) decomposition $\mathfrak{g}_{0} \oplus \mathbf{V}_{c}$. Then, the curvature coefficients of the lattice canonical connection in the frame $v_{i}, i=1, \ldots, n$, are "almost" identical to the directional derivatives of the torsion coefficients of the lattice connection (18) as

$$
\widehat{R}_{j k l}^{m}+\widehat{R}_{k l j}^{m}+\widehat{R}_{l j k}^{m}=\left[T_{j k, l}^{m}+T_{k l, j}^{m}+T_{l j, k}^{m}\right]\left(p_{0}\right) .
$$

Realize that although the vanishing of the directional derivatives of the torsion $T_{j k}^{i}$ forces the curvature $\widehat{R}_{j k l}^{i}$ to vanish by construction, the reverse is not necessarily true. Indeed, when the canonical decomposition $\mathfrak{g}_{0} \oplus \mathbf{V}_{c}$ is a semi-direct product of Lie subalgebras, the curvature of the canonical linear connection vanishes as $\mathbf{V}_{c}$ is an ideal in $\mathfrak{g}$, despite the fact that the coefficients $T_{j k}^{i}$ may still be position dependent.

Example 4. Consider a defective kinematic state of $U=\left\{(x, y, z) \in \mathbb{R}^{3}: x y \neq 0\right\}$ defined by a lattice $\mathfrak{l}$ given by three linearly independent smooth vector fields $l_{i}: U \rightarrow \mathbb{R}^{3}, i=1,2,3$, such that

$$
l_{1}=\mathbf{e}_{1}, \quad l_{2}=\mathbf{e}_{2}-y \mathbf{e}_{1}, \quad l_{3}=x \mathbf{e}_{1}+y \mathbf{e}_{2}+\mathbf{e}_{3},
$$

where we use the standard Cartesian coordinate system in $\mathbb{R}^{3}$ and where the vectors $\mathbf{e}_{i}, i=1,2,3$, denote the corresponding standard basis. The vector fields $l_{i}, i=1,2,3$, form a four-dimensional lattice algebra $\mathbf{L}$. Indeed, calculating Lie brackets of the given vector fields we obtain that

$$
\left[l_{1}, l_{2}\right]=0, \quad\left[l_{1}, l_{3}\right]=l_{1}, \quad\left[l_{2}, l_{3}\right]=\mathbf{e}_{2}=l_{4},
$$

whereas

$$
\left[l_{1}, l_{4}\right]=0, \quad\left[l_{2}, l_{4}\right]=l_{1}, \quad\left[l_{3}, l_{4}\right]=-l_{4} .
$$

This also shows that the only non-vanishing components of the torsion of the lattice connection induced by the frame $\left\{l_{1}, l_{2}, l_{3}\right\}$ are

$$
T_{13}^{1}=T_{23}^{2}=1, \quad T_{23}^{1}=y .
$$

Each generator $l_{j}, j=1, \ldots, 4$, of the lattice algebra $\mathbf{L}$ induces a one-parameter group of (smooth) transformations of $U$ superposition of which provides a (left) action (1) of a four-parameter group $G=\{(a, b, c, d)$ : $a, b, c, d \in \mathbb{R}\}$ such that

$$
\phi((a, b, c, d),(x, y, z))=\left((x+a-y b) e^{d},(y+b+c) e^{d}, z+d\right),
$$

where the group multiplication in $G$ takes the form

$$
g \bar{g}=\left(\bar{a}+a e^{-\bar{d}}-b(\bar{b}+\bar{c}), b+\bar{b},(b+c) e^{-\bar{d}}+\bar{c}-b, d+\bar{d}\right)
$$

for any pair $g, \bar{g} \in G$.

Selecting a point $p_{0}=\left(x_{0}, y_{0}, z_{0}\right) \in U$, the isotropy group of the action $\phi$ at $p_{0}$ is a one-parameter subgroup $G_{0}$ of $G$ such that

$$
G_{0}=\left\{\left(y_{0} b, b,-b, 0\right): b \in \mathbb{R}\right\} .
$$

Viewing the group multiplication in $G$ as the action of a group on itself, we can determine the left- and the rightinvariant vector fields on $G$ by considering two tangent maps: $d_{e} L_{g}: T_{e} G \rightarrow T_{g} G$ and $d_{e} R_{g}: T_{e} G \rightarrow T_{g} G$, where $L_{g}$ and $R_{g}$ represent the left and the right translation of $G$ by an element $g \in G$, respectively. Thus, the multiplication rule in $G$ implies that

$$
d_{e} L_{g}=\left(\begin{array}{cccc}
1 & -b & -b & -a \\
0 & 1 & 0 & 0 \\
0 & 0 & 1 & -(b+c) \\
0 & 0 & 0 & 1
\end{array}\right)
$$

and

$$
d_{e} R_{g}=\left(\begin{array}{cccc}
e^{-d} & -(b+c) & 0 & 0 \\
0 & 1 & 0 & 0 \\
0 & e^{-d}-1 & e^{-d} & 0 \\
0 & 0 & 0 & 1
\end{array}\right)
$$


where $e=(0,0,0,0)$ is a unit element. This shows that the space of all left-invariant vector fields on $G$ is spanned by

$$
\begin{aligned}
& \mathbf{v}_{1}=\mathbf{g}_{1}, \mathbf{v}_{2}=-b \mathbf{g}_{1}+\mathbf{g}_{2}, \\
& \mathbf{v}_{3}=-b \mathbf{g}_{1}+\mathbf{g}_{3}, \mathbf{v}_{4}=-a \mathbf{g}_{1}-(b+c) \mathbf{g}_{3}+\mathbf{g}_{4},
\end{aligned}
$$

whereas the space of all right-invariant vector fields on $G$ is spanned by

$$
\begin{array}{ll}
\mathbf{w}_{1}=e^{-d} \mathbf{g}_{1}, & \mathbf{w}_{2}=-(b+c) \mathbf{g}_{1}+\mathbf{g}_{2}+\left(e^{-d}-1\right) \mathbf{g}_{3}, \\
\mathbf{w}_{3}=e^{-d} \mathbf{g}_{3}, & \mathbf{w}_{4}=\mathbf{g}_{4},
\end{array}
$$

where $\mathbf{g}_{1}, \ldots, \mathbf{g}_{4}$ denote the standard basis on $G$. It is easy to check that they are isomorphic Lie algebras and that they are isomorphic to the lattice algebra $\mathbf{L}$. Moreover, one can show that the basis $\left\{v_{1}, v_{2}, v_{3}, v_{4}\right\}$ is equivalent (via the inverse map in $G$ ) to the right-invariant basis $\left\{w_{1}, w_{2}, w_{3}, w_{4}\right\}$.

Consider the orbit map $\phi_{p_{0}}: G \rightarrow U$. Its tangent map $d_{g} \phi_{p_{0}}: T_{g} G \rightarrow T_{\phi_{p_{0}}(g)} U$ establishes an isomorphism between the right Lie algebra of $G$ and the lattice algebra $\mathbf{L}$. Indeed, given the group action (45)

$$
d_{g} \phi_{p_{0}}=\left(\begin{array}{cccc}
e^{d} & -y_{0} e^{d} & 0 & \left(x_{0}+a-y_{0} b\right) e^{d} \\
0 & e^{d} & e^{d} & \left(y_{0}+b+c\right) e^{d} \\
0 & 0 & 0 & 1
\end{array}\right) .
$$

Thus,

$$
d_{g} \phi_{p_{0}}\left(\begin{array}{cccc}
e^{-d} & -(b+c) & 0 & 0 \\
0 & 1 & 0 & 0 \\
0 & e^{-d}-1 & e^{-d} & 0 \\
0 & 0 & 0 & 1
\end{array}\right)=\left(\begin{array}{cccc}
1 & -y & 0 & x \\
0 & 1 & 1 & y \\
0 & 0 & 0 & 1
\end{array}\right)
$$

where $(x, y, z)=\phi\left(g, p_{0}\right)=\left(\left(x_{0}+a-y_{0} b\right) e^{d},\left(y_{0}+b+c\right) e^{d}, z_{0}+d\right)$.

This shows that the vector space $\mathbf{V}_{c}=\operatorname{span}\left\{\mathbf{v}_{1}, \mathbf{v}_{2}, \mathbf{v}_{4}\right\}$ is indeed a canonical vector complement to the isotropy Lie subalgebra $\mathfrak{g}_{0}=\operatorname{span}\left\{v_{0}\right\}=\operatorname{span}\left\{\left(y_{0}, 1,-1,0\right)\right\}$ in $\mathfrak{g}$. Moreover, the decomposition $\mathfrak{g}_{0} \oplus \mathbf{V}_{c}$ is reductive as

$$
\left[\mathbf{v}_{0}, \mathbf{v}_{1}\right]=0, \quad\left[\mathbf{v}_{0}, \mathbf{v}_{2}\right]=-\mathbf{v}_{1},\left[\mathbf{v}_{0}, \mathbf{v}_{4}\right]=-y_{0} \mathbf{v}_{1} .
$$

Hence, the lattice canonical connection exists. Relabeling the spanning set of $\mathbf{V}_{c}$ and calculating the relevant Lie brackets we obtain that the torsion $\widehat{T}_{j k}^{i}$ has only three non-zero components

$$
\widehat{T}_{13}^{1}=\widehat{T}_{23}^{2}=-1 \text { and } \widehat{T}_{23}^{1}=-y_{0},
$$

whereas the most curvature coefficients $\widehat{R}_{j k l}^{i}$ vanish except

$$
\widehat{R}_{232}^{1}=-1 \text { and } \widehat{R}_{233}^{1}=-y_{0} .
$$

Example 5. In our final example, we revisit (Example 1) the lattice

$$
\mathfrak{l}=\left\{l_{1}, l_{2}\right\}=\left\{\mathbf{e}_{1},-x \mathbf{e}_{2}\right\} .
$$

The left Lie algebra $\mathfrak{g}$ of the group $G$ induced by $\mathfrak{l}$ is spanned by

$$
\mathbf{v}_{1}=\mathbf{g}_{1}, \quad \mathbf{v}_{2}=\mathbf{g}_{2}-a \mathbf{g}_{3}, \quad \mathbf{v}_{3}=\mathbf{g}_{3},
$$

whereas its right algebra is generated by

$$
\mathbf{w}_{1}=\mathbf{g}_{1}-b \mathbf{g}_{3}, \quad \mathbf{w}_{2}=\mathbf{g}_{2}, \quad \mathbf{w}_{3}=\mathbf{g}_{3} .
$$

Knowing that the tangent to the orbit map at a point $p_{0} \in U$ evaluated at the identity of $G$ is

$$
d_{e} \phi_{p_{0}}=\left(\begin{array}{ccc}
1 & 0 & 0 \\
0 & x_{0} & -1
\end{array}\right)
$$


it should be easy to see that the corresponding canonical vector space $\mathbf{V}_{c}=\operatorname{span}\left\{\mathbf{v}_{1}, \mathbf{v}_{2}\right\}$ and that $\mathfrak{g}=\mathfrak{g}_{0} \oplus \mathbf{V}_{c}$ where the isotropy algebra $\mathfrak{g}_{0}=\operatorname{span}\left\{\mathbf{v}_{0}\right\}=\operatorname{span}\left\{\mathbf{g}_{2}-\left(a+x_{0}\right) \mathbf{g}_{3}\right\}$. Unfortunately, it should also be easy to see that such a decomposition is not reductive as

$$
\left[\mathbf{v}_{1}, \mathbf{v}_{0}\right]=-\mathbf{g}_{3} \notin \mathbf{V}_{c} .
$$

\section{Notes}

1. A curvature is often associated with a presence of disclinations [4].

2. In other publications on this topic, we use also the term lattice frame.

3. Our presentation is mathematically correct in any finite dimension $n$.

4. Note that not every lattice $\mathfrak{l}$ defines a finite-dimensional Lie algebra $\mathbf{L}$. For example, a tree trunk dislocation, defined on $\mathbb{R}^{2}-$ $\{0\}$ by unit vector fields $\frac{\partial}{\partial r}, \frac{1}{r} \frac{\partial}{\partial \theta}$ associated with the polar coordinate system $r, \theta$, induces, as it is easy to check, an infinitedimensional Lie algebra of vector fields (Epstein, M. Private communication, 2021).

5. The left and right Lie algebras of any Lie group $G$ are isomorphic. The isomorphism is established by the inverse map $i: G \rightarrow G$, i.e., $\mathfrak{i}(g)=g^{-1}$, where $g \in G$.

6. Looking back at the lattice $\mathfrak{l}$ from Example 1, we can easily show that the only non-zero torsion coefficient is $T_{12}^{2}=\frac{1}{x}$. Viewing this lattice as immersed in $\mathbb{R}^{3}$, that is, considering a lattice

$$
\mathfrak{l}=\left\{l_{1}, l_{2}, l_{3}\right\}=\left\{\mathbf{e}_{1},-x \mathbf{e}_{2}, \mathbf{e}_{3}\right\},
$$

one can show that the only non-vanishing component of the dislocation density tensor is $S_{23}=-\frac{1}{x}$.

7. As we pointed out earlier, not every lattice $\mathfrak{l}$ induces a finite-dimensional Lie algebra $\mathbf{L}$. Thus, by assuming that it does we restrict the choice of the kinematic states we are able to analyze using this approach.

8. Note that the assumption that the homogeneous space admits a reductive decomposition is essential for the existence of a canonical connection as there are non-reductive homogeneous spaces which do not admit any invariant affine connection (Bryant, R. Private communication, 2021.). On the other hand, every reductive homogeneous space admits a (left-invariant) canonical connection [16].

9. This approach was first proposed in [5].

\section{Funding}

This research received no specific grant from any funding agency in the public, commercial, or not-for-profit sectors.

\section{ORCID iD}

Marek Elzanowski iD https://orcid.org/0000-0003-0494-6496

\section{References}

[1] Davini, C. A proposal for a continuum theory of defective crystals. Arch Rat Mech Anal 1986; 96: $295-317$.

[2] Parry, GP. Group properties of defective crystal structures. Math Mech Solids 2003; 8: 515-538.

[3] Elżanowski, M, and Preston, S. On continuously defective elastic crystals. Miskolc Math Notes 2013; 14(2): 659-670.

[4] Yavari, A, and Goriely, A. Riemann-Cartan geometry of nonlinear disclination mechanics. Math Mech Solids 2013; 18(1): 91-102.

[5] Elżanowski, M. Torsion and curvature in continuously defective solid crystals. Atti Accad Pelor Pericol Classe Sci Fis Mat Nat 2021; 99(11) A4: 1-9.

[6] Elżanowski, M, and Parry, GP. Connection and curvature in crystals with non-constant dislocation density. Math Mech Solids 2019; 24(6): 1714-1725.

[7] Kobayashi, S. Transformation Groups in Differential Geometry. Berlin: Springer-Verlag, 1995.

[8] Olver, PJ. Equivalence, Invariants, and Symmetry. Cambridge: Cambridge University Press, 1995.

[9] Kobayashi, S, and Nomizu, K. Foundations of Differential Geometry, Vols. I and II. New York: John Wiley \& Sons, Inc., 1996.

[10] Elżanowski, M, and Parry, GP. A kinematics of defects in solid crystals. In: Segev,R and Epstein, M (eds.) Geometric Continuum Mechanics. Cham: Birkhäuser, 2020.

[11] Epstein, M. The Geometrical Language of Continuum Mechanics. Cambridge: Cambridge University Press, 2010.

[12] Lee, EH. Elastic-plastic deformation at finite strains. J Appl Mech 1969; 36: 1-6.

[13] Parry, GP, and Šilhavý, M. Elastic invariants in the theory of defective crystals. Proc R Soc Lond A 1999; 455: $4333-4346$.

[14] Elżanowski, M, and Parry, GP. Material symmetry in a theory of continuously defective crystals. J Elasticity 2004; $74: 215-237$.

[15] Poor, WA. Differential Geometric Structures. New York: McGraw-Hill, 1981.

[16] Wang, HC. On invariant connections over a principal fiber bundle. Nagoya Math J 1958; 13: 1-19. 